\section{D-coupling multiple myeloma's codependents}

\section{By Michael J. Haas, Senior Writer}

Three years ago researchers at the Mayo Clinic Arizona reported that most types of multiple myeloma overexpress cyclin D1 and/or cyclin D2. ${ }^{1}$ Following on that earlier work, a new research team at the clinic has explored whether these proteins are viable targets for treating the disease-and if so, how to target them.

In the Journal of Clinical Investigation, the researchers reported that kinetin riboside, a naturally occurring cytokinin, inhibited multiple myeloma (MM) cell proliferation by suppressing the expression of cyclins D1 and D2 (CCND1; CCND2). The group suggests that derivatives of kinetin riboside could be more selective and less toxic to normal cells than current treatments for MM. ${ }^{2}$

Both the researchers and company representatives contacted by $S c i B X$ said kinetin riboside derivatives could be important additions to the MM armamentarium, though pharmacokinetic improvements are needed because kinetin riboside itself has a short half-life.

The research team, led by Keith Stewart, professor of medicine at the Mayo Clinic Arizona, also included researchers from the Ontario Cancer Institute at Princess Margaret Hospital, the McLaughlin Centre for Molecular Medicine and Mount Sinai Hospital in Toronto.

The three D cyclins, CCND1, CCND2 and cyclin D3 (CCND3), play a role in regulating cell division and the progression of cells through the growth phase (G0/G1) of the cell cycle. In normal cells, the functions of all three proteins are redundant and interchangeable. But in most types of myeloma, either CCND1, CCND2 or both are overexpressed, and MM cells depend on the upregulated cyclin(s) for survival.

"It's clear that many tumors overcome the constraints of the G0/G1 checkpoint by overexpressing a cyclin $\mathrm{D}$ gene, and that cyclin Ds are amongst the most commonly overexpressed proteins in human cancers," said Rodger Tiedemann, postdoctoral fellow in research at the Mayo Clinic Arizona and research team spokesperson.

The deregulation of cyclin $\mathrm{D}$ genes and the loss of normal cyclin $\mathrm{D}$ redundancy in MM and other cancers is what led the researchers to seek compounds that target the expression of those genes, he said.

As a first step, the team showed that silencing the expression of
CCND1 and CCND2 genes with small hairpin RNA induces cell-cycle arrest in MM cell lines, thus confirming the dependence of the cells on one or both of the cyclins.

Next, the team screened compound libraries from Sigma-Aldrich Co., Prestwick Chemical Inc. and MicroSource Discovery Systems Inc. for inhibitors of CCND2 expression. The team chose this gene for the screen because it is more frequently overexpressed than CCND1 in poor-prognosis types of MM.

Kinetin riboside-a cytokinin found in coconut milk-emerged as the most promising lead.

In MM cell lines and in bone marrow cells from MM patients, kinetin riboside suppressed the expression of CCND1 and CCND2. The proposed mechanism is that kinetin riboside induces upregulation of several repressors that bind to and block expression of the two genes. The repressors are isoforms of one another and are coded by the $C A M P$ response element modulator (CREM) gene, which is involved in the regulation of transcription factors.

Kinetin riboside does not suppress expression of CCND3 because that gene does not have the same cAMP response element-the region of a gene that allows its expression to be promoted or suppressed-as CCND1 and CCND2, the researchers wrote.

"MM cells are especially susceptible to repression of cyclins D1 and D2," Tiedemann said. "In normal cells cyclin D3 function could compensate for this repression," but cancer cells cannot do this as readily because of their dependence on CCND1 and/or CCND2.

Kinetin riboside also showed in vitro activity against other cancers known to have deregulated cyclin $\mathrm{D}$ genes, including certain types of breast cancer, pancreatic cancer, melanoma, glioma and non-Hodgkin's lymphoma (NHL).

"Although some of these tumor lines were refractory to kinetin riboside, others were more responsive than myeloma tumor lines," Tiedemann said.

Lastly, the team showed kinetin riboside prevents the growth of MM tumors in xenograft mouse models.

"The discovery of kinetin riboside as a lead compound is quite exciting," said Andrew Mazar, CSO of Attenuon LLC. "The researchers did a really good job of validating the target and elucidating the mechanism" and their activity experiments in human bone marrow cells provide some early toxicity information as well, he said.

Attenuon's ATN-224, a second-generation version of tetrathiomolybdate that targets superoxide dismutase1 (SOD1) mRNA, is in Phase II testing for advanced melanoma, breast cancer and relapsed prostate cancer patients who have never had hormonal therapy, and it is in Phase I/II testing for MM.

Mazar also said it was impressive that the team's screen yielded a molecule that directly regulates the expression of D cyclins instead of an upstream signaling pathway. "Small molecules that target signaling pathways-such as kinase inhibitors-may indeed affect signaling, but 
this is usually a by-product of the pathway that they inhibit," he said. "Hits as good as kinetin riboside are not generally discovered with an assay that looks at expression of genes of interest."

\section{Good lead hunting}

The catch to the positive mouse data is that researchers had to dose the mice 5 times daily because of kinetin riboside's 15-minute half-life. As a result, both Stewart and Tiedemann said the cytokinin itself does not have an adequate pharmacokinetic profile to be a drug candidate.

The good news, said Tiedemann is that "kinetin riboside is a purine analog, and these analogs have already been successful as drugs. So the structural core of kinetin riboside is amenable to drug development."

Mazar said the compound's poor pharmacokinetic profile indicates a need for further optimization but agreed that the core purine structure is highly amenable to making derivatives.

"Also, the animals showed good tolerance to the high dosing" necessitated by kinetin riboside's short half-life, indicating that the compound was not generally toxic, he said.

"The biology is clear," said Daniel Adelman, SVP of development and CMO of Sunesis Pharmaceuticals Inc. "D cyclins are relevant and important targets in multiple myeloma-but not the only ones and not necessarily the most important ones. Targeting CCND1 and CCND2, along with cyclin-dependent kinases, is likely to be complementary, not competitive."

Sunesis' SNS-032, an inhibitor of cyclin-dependent kinases 2, 7 and 9 (CDK2; CDK7; CDK9) is in Phase I trials for MM and chronic lymphocytic leukemia (CLL). The company's lead compound is SNS595, a cell-cycle and topoisomerase II inhibitor that is in a Phase II trial to treat ovarian cancer and a Phase Ib trial to treat relapsed or refractory acute myeloid leukemia (AML).

Timothy Clackson, SVP and CSO of Ariad Pharmaceuticals Inc., agreed that kinetin riboside was a promising lead because the purine core is a known pharmacophore. "Different structures added to the purine core would make it more drug-like and improve its potency, pharmacokinetics and other drug properties," he told SciBX.

The screen Stewart's team performed "wouldn't be expected to give a good drug on the first pass," he added. "The issue now is finding a molecule that works like this one but has a better pharmacokinetic profile."

Clackson noted that purine analogs have been the basis for other therapeutics, including Ariad's AP23464, a dual Src-Abl kinase inhibitor for which development was discontinued in 2004 for reasons unrelated to the purine-based structure. ${ }^{3}$

Ariad's AP24534, an inhibitor of multiple kinases including BclAbl tyrosine kinase, is in a Phase I trial to treat CML, and the company expects to begin a Phase I trial in solid tumors this year. Ariad has not disclosed the structure of AP24534, but Clackson said it is not a purine analog.

Stewart's research team is now synthesizing kinetin riboside derivatives and looking for analogs with longer half-lives.

"Our main focus has been on improving the pharmacokinetics, by developing analogs and testing these on cell lines," he said. "We have identified some candidates."

Tiedemann noted that "there is certainly potential for kinetin riboside derivatives to have activity against other cancers beyond multiple myeloma. In developing derivatives, we are taking a broad interest in potential activities of derivatives against a spectrum of tumor types."

Stewart said the Mayo Clinic and the University Health Network in Toronto have filed a provisional patent on the use of kinetin riboside to treat cancer. The compound itself is already commercially available. The free base, kinetin, is used in some topical cosmetics.

Stewart said the team would welcome commercial partnerships to further its exploration of kinetin riboside derivatives.

\section{REFERENCES}

1. Bergsagel, P. et al. Blood 106, 296-303 (2005)

2. Tiedemann, R. et al. J. Clin. Invest.; published online April 22, 2008; doi:10.1172/JCl34149

Contact: A. Keith Stewart, Mayo Clinic, Scottsdale, Ariz. e-mail: stewart.keith@mayo.edu

3. Haan, K. BioCentury 12(37), A1-A4; Aug. 23, 2004

COMPANIES AND INSTITUTIONS MENTIONED

Ariad Pharmaceuticals Inc. (NASDAQ:ARIA), Cambridge, Mass.

Attenuon LLC, San Diego, Calif.

Mayo Clinic Arizona, Scottsdale, Ariz.

McLaughlin Centre for Molecular Medicine, Toronto, Ontario, Canada

MicroSource Discovery Systems Inc., Gaylordsville, Conn.

Mount Sinai Hospital, Toronto, Ontario, Canada

Prestwick Chemical Inc., Illkirch, France

Princess Margaret Hospital, Toronto, Ontario, Canada

Sigma-Aldrich Co. (NASDAQ:SIAL), St. Louis, Mo.

Sunesis Pharmaceuticals Inc. (NASDAQ:SNSS), South San Francisco, Calif.

University Health Network, Toronto, Ontario, Canada 